\title{
Entanglement frustration for Gaussian states on symmetric graphs
}

\author{
M.M. Wolf, F. Verstraete, and J.I. Cirac \\ Max-Planck-Institut für Quantenoptik, Hans-Kopfermann-Str. 1, Garching, D-85748, Germany
}

(Dated: November 29, 2018)

\begin{abstract}
We investigate the entanglement properties of multi-mode Gaussian states, which have some symmetry with respect to the ordering of the modes. We show how the symmetry constraints the entanglement between two modes of the system. In particular, we determine the maximal entanglement of formation that can be achieved in symmetric graphs like chains, $2 d$ and $3 d$ lattices, mean field models and the platonic solids. The maximal entanglement is always attained for the ground state of a particular quadratic Hamiltonian. The latter thus yields the maximal entanglement among all quadratic Hamiltonians having the considered symmetry.
\end{abstract}

Classically as well as quantum mechanically the global ordering or symmetry of a system often imposes highly non-trivial constraints on its local properties. These kinds of frustration effects lie at the heart of ordered interacting systems, and physicists are faced with these phenomena whenever dealing with lattice systems or molecular structures. The present paper is devoted to investigate how the entanglement of two subsystems of a larger system is constraint by such a global symmetry for some particularly interesting class of states, the so-called Gaussian states [1].

Gaussian states appear very naturally in several branches of physics where entanglement plays a predominant role. The electromagnetic field in most quantum optical setups, atomic ensembles interacting with such fields 2], the motion of a collection of trapped ions, or the low energy (bosonic) excitations of many interacting systems can be very well described by these states. This is due to the fact that quantum field theories can be, in some regimes, approximated by Hamiltonians which are quadratic in some bosonic operators, and thus in thermal equilibrium as well as a result of the dynamics the corresponding states are Gaussian. Thus, there is a growing interest in understanding the entanglement properties of these states [2, 3, 4, 5, 6, 7].

Our results quantify a very intuitive property of entanglement, which distinguishes it from the usual correlations found in classical systems: one particle can share entanglement only with a limited number of other particles [8], which in turn becomes smaller and smaller as the amount of entanglement increases. Furthermore, the entanglement that can be shared by a subset of particles strongly depends on the symmetries of the multiparticle state. For example, if we have a set of particles distributed on a lattice in a state with translational symmetry, the maximal entanglement between any two nearest neighboring particles should depend on the number of spatial dimensions, and should decrease if the total number of particles increases. For one-dimensional rings of spin $\frac{1}{2}$ particles quantitative investigations of this kind were started in [9]. However, the involved optimization problems are highly non-trivial such that up to now only a lower bound for the achievable Entanglement of Forma- tion $(\mathrm{EoF})[10]$ is known. In the case of Gaussian states, the situation can become even more intriguing since for two modes only, the amount of entanglement becomes unbounded. If we consider three modes, and impose that the global state is invariant under permutations, it turns out that the maximum EoF between any pair of modes becomes finite. By increasing the number of modes and imposing different symmetries to the global state, this quantity experiences strong modifications. In this work we determine the Gaussian state of $N$ modes which gives rise to the maximal EoF between a selected pair of modes, for any $N$ and a large variety of symmetry groups.

From our analysis it also follows that the state for which the maximum entanglement is generated under a given symmetry corresponds to the ground state of a particular Hamiltonian, quadratic in the bosonic operators, which can be easily constructed. Thus we can determine the Hamiltonian, invariant under a certain symmetry group, that generates the maximum two-mode entanglement for the physical systems mentioned above.

Although we will consider rather general symmetry groups, we will illustrate our results for groups which can be associated to symmetric graphs (Fig प(a)), since they give an intuitive geometric depiction of the group and they are the ones that naturally appear in many physical systems. For example, we will give the optimal EoF for states that have the symmetries of a lattice in any dimension, including square, cubic, hexagonal, and trigonal lattices (Tab【), or those of all platonic solids (Tab!II).

Let $\left(Q_{1}, \ldots, Q_{N}, P_{1}, \ldots, P_{N}\right):=R$ be the $N$ conjugate pairs of canonical operators characterizing $N$ modes and obeying the canonical commutation relations $\left[R_{k}, R_{l}\right]=$ $i \sigma_{k l}$ with $\sigma$ being the symplectic matrix (cf.[1]). Let us consider a subgroup $G$ of the permutation group and two particular modes, $k, l \leq N$, for which there exists a group element such that $g(k)=l$ and $g(l)=k$ (this condition will be relaxed later on). We construct a Hamiltonian operator as follows

$$
\hat{H}_{\text {max }}=\frac{1}{4|G|} \sum_{g \in G}\left(Q_{g(k)}+Q_{g(l)}\right)^{2}+\left(P_{g(k)}-P_{g(l)}\right)^{2}(1)
$$

Let us denote by $E_{0}$ the ground state energy and by 


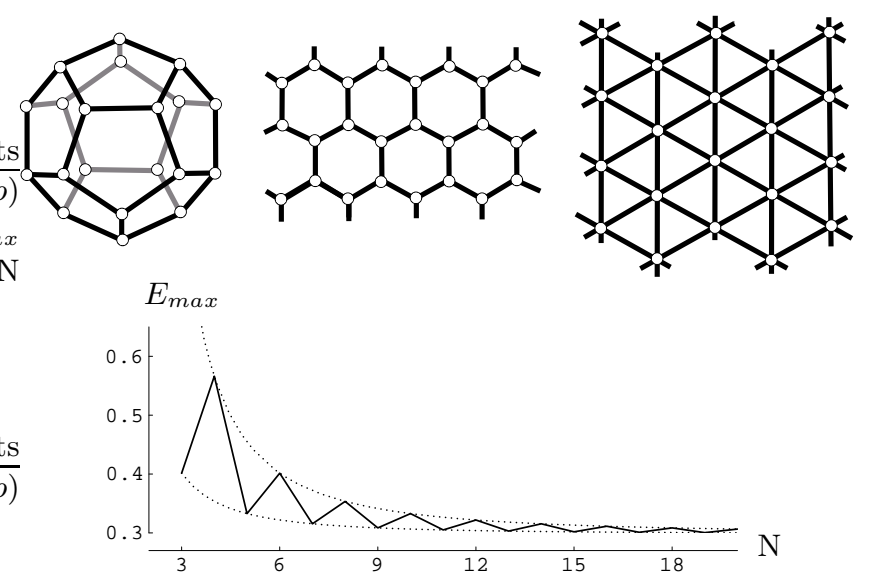

FIG. 1: (a): Apart from chains, cubic lattices and meanfield clusters there are several familiar symmetric graphs. Examples are the five platonic solids (e.g. the dodecahedron), and hexagonal or trigonal lattices. (b): Maximal nearest neighbor entanglement $E_{\max }$ (ebits) in a ring of $N$ harmonic oscillators. The dotted curves represent the envelopes corresponding to Eq. 10.

$\Psi_{0}$ the corresponding ground state, which is a Gaussian state, i.e., $\Psi_{0}$ has a Gaussian Wigner function. The relation between this Hamiltonian and the EoF will later on be established by linearizing the expression for the latter. We will show that the Gaussian state which is invariant under $G$ and which maximizes the EoF of the modes $k$ and $l$ is exactly $\Psi_{0}$ and that the corresponding EoF is $E_{\max }=E_{F}\left(E_{0}\right)$, where

$$
E_{F}(\Delta)=c_{+}(\Delta) \log \left[c_{+}(\Delta)\right]-c_{-}(\Delta) \log \left[c_{-}(\Delta)\right]
$$

and $c_{ \pm}(\Delta):=\left(\Delta^{-1 / 2} \pm \Delta^{1 / 2}\right)^{2} / 4$. Hence, the ground state of $\hat{H}_{\max }$ has maximum entanglement under all $G$-invariant Gaussian states and conversely, among all quadratic $G$-invariant Hamiltonians $\hat{H}_{\max }$ generates the largest amount of entanglement at zero temperature.

The ground state of Hamiltonians of the form (11) can be easily determined by resorting to symplectic space. We define the covariance matrix (CM) of a Gaussian state $\rho$ as usual,

$$
\Gamma_{k l}:=\left\langle\left\{\left(R_{k}-\left\langle R_{k}\right\rangle\right),\left(R_{k}-\left\langle R_{k}\right\rangle\right)\right\}_{+}\right\rangle,
$$

which must fulfill $\Gamma \geq i \sigma$ [1]. Let us also introduce the Hamiltonian matrix [5] corresponding to $\hat{H}_{\max }$ as $H=$ $H_{+} \oplus H_{-}$with [13]

$$
\begin{aligned}
H_{ \pm} & :=\frac{1}{|G|} \sum_{g \in G} T_{g} h_{ \pm}^{(k, l)} T_{g}^{-1} \\
h_{ \pm}^{(k, l)} & :=\frac{1}{4}[|k\rangle\langle k|+| l\rangle\langle l| \pm(|k\rangle\langle l|+| l\rangle\langle k|)] .
\end{aligned}
$$

The matrix $H \geq 0$ can be diagonalized by a symplectic matrix $S, H=S D S^{T}$. Since $\operatorname{tr}[\rho \hat{H}]=\operatorname{tr}[\Gamma H]$, the

\begin{tabular}{|l|c|c|}
\hline lattice & $E_{\max }$ & $N_{a}$ \\
\hline hexagonal (2d) & 10.61 & 3 \\
square (2d) & 6.31 & 4 \\
trigonal (2d) & 2.69 & 6 \\
cubic (3d) & 2.62 & 6 \\
\hline
\end{tabular}

TABLE I: Maximal nearest neighbor entanglement $E_{\max }$ (in units of $10^{-2}$ ebits) for some infinite $2 d$ and $3 d$ lattices. $N_{a}$ is the number of adjacent vertices.

ground state energy of $\hat{H}$ is given by 14 ]

$$
E_{0}=\inf _{\Gamma} \operatorname{tr}[\Gamma H]=\inf _{\Gamma} \operatorname{tr}[\Gamma D]=2\left\|\sqrt{H_{+}^{1 / 2} H_{-} H_{+}^{1 / 2}}\right\|_{1} .
$$

Since $H$ has a null space, the $\mathrm{CM} \Gamma_{0}$ of the ground state $\Psi_{0}$ of $\hat{H}$ is achieved in the limit $\epsilon \rightarrow 0$ of $\Gamma_{\epsilon}:=S_{\epsilon}^{-1 T} S_{\epsilon}^{-1}$, where $S_{\epsilon}$ is the symplectic matrix that diagonalizes $H+$ $\epsilon 1$.

Before we prove the above statements, let us utilize the results to analyze the maximum EoF for several interesting symmetry groups. We will concentrate on groups which can be associated to a symmetric graph.

Consider a simple undirected graph with $N$ vertices, characterized by an adjacency matrix $A$, which is such that $A_{k l}=1$ if the vertices $k$ and $l$ are connected by an edge, and $A_{k l}=0$ if there is either no edge or $k=l$. The symmetry group $G$ of the graph contains all permutations $g$, which commute with the adjacency matrix $[A, g]=0$. The graph is called symmetric if all edges as well as all vertices are equal in the sense that every edge and every vertex can be mapped onto every other one by an element of $G$. Examples of symmetric graphs are given in Fig 1(a) and TabIII By utilizing this symmetry we can simplify

$$
H_{ \pm}=\frac{1}{|\mathcal{E}|} \sum_{(k, l) \in \mathcal{E}} h_{ \pm}^{(k, l)}
$$

where $\mathcal{E}=\left\{(k, l) \mid A_{k l}=1\right\}$ is the set of edges. Hence, the sum in Eq.(1) runs over all edges which correspond therefore to physical interactions between the adjacent modes. By observing that $\left[H_{+}, H_{-}\right]=0$ we can derive the ground state $\mathrm{CM}$ (or the respective limit):

$$
\Gamma_{0}=\sqrt{H_{-} H_{+}^{-1}} \oplus \sqrt{H_{+} H_{-}^{-1}} .
$$

Note that when acting on two modes only, the ground state of $\hat{H}_{\text {max }}$ is the original singular EPR-state. Moreover, since $H_{-}$has a Kernel containing the vector $(1,1, \ldots, 1)$ the ground state of any $\hat{H}_{\max }$ will always be singular and the maximal entanglement is thus only attained exactly in the limit of infinite squeezing.

In the following we will apply the obtained results to some examples of familiar symmetric graphs.

Chains and rings: The simplest non-trivial example of a symmetric graph is a ring of $N$ nodes representing translation and reflection symmetry. In this case the 
operators $H_{ \pm}$have the form

$$
H_{ \pm}=\frac{1}{4 N}\left[21 \pm\left(T+T^{-1}\right)\right]
$$

where $T_{k l}=\delta_{k, l+1 \bmod N}$ is the cyclic shift operator. $H_{ \pm}$ are so-called circulant matrices [1], which can be diagonalized simultaneously by a Fourier transform yielding

$$
E_{0}=\frac{1}{N} \sum_{l=0}^{N-1}\left|\sin \frac{2 \pi}{N} l\right|=\left\{\begin{array}{cc}
\frac{2}{N} \cot \frac{\pi}{N}, & N \text { even } \\
\frac{1}{N} \cot \frac{\pi}{2 N}, & N \text { odd }
\end{array} .\right.
$$

Hence, the entanglement remains finite and is suppressed in rings with an odd number of modes (see Fig (1). It approaches $E_{\max }=0.30$ ebits $\left(E_{0}=\frac{2}{\pi}\right)$ in the limit $N \rightarrow \infty$. This value is comparable to the 0.29 ebits conjectured for an infinite chain of spin $\frac{1}{2}$ particles $[9]$.

Cubic lattices: The result obtained for the chain has a straight forward extension to $d$-dimensional cubic lattices. Imposing periodic boundary conditions (i.e. a lattice on a torus) for a cubic lattice of $N^{d}$ modes we get

$$
H_{ \pm}=\frac{1}{4 N^{d}}\left[2 \mathbf{1} \pm \frac{1}{d} \sum_{a=1}^{d}\left(T_{(a)}+T_{(a)}^{-1}\right)\right]
$$

where now $T_{(a)}$ is the shift operator acting on the $a^{\prime}$ th of $d$ tensor factors, each corresponding to one of the dimensions of the lattice (e.g. $T_{(2)}=\mathbf{1} \otimes T \otimes \mathbf{1} \ldots$ ). Diagonalizing $H_{ \pm}$by a tensor product of Fourier transforms leads to

$$
E_{0}=\frac{1}{N^{d}} \sum_{l_{1}=1}^{N} \ldots \sum_{l_{d}=1}^{N}\left[1-\frac{1}{d^{2}}\left(\sum_{k=1}^{d} \cos \frac{2 \pi}{N} l_{k}\right)^{2}\right]^{\frac{1}{2}}
$$

which goes to $1\left(E_{\max } \rightarrow 0\right)$ for $d \rightarrow \infty$, and is calculated explicitly in Tab】 for the infinite two and three dimensional cubic lattice.

Mean field clusters: When every mode is connected to every other one, i.e., when we have complete permutation symmetry, then

$$
H_{ \pm}=[2 N(N-1)]^{-1}[(N-1) \mathbf{1} \pm(\mathbf{E}-\mathbf{1})],
$$

where $\mathbf{E}_{k l}=1$, which leads to $E_{0}=\sqrt{\frac{N-2}{N}}$. Hence, the maximal entanglement decreases with the number $N$ of modes and vanishes as $\sim \frac{1}{N^{2}} \log N$ in the limit $N \rightarrow \infty$.

Platonic solids: The results for the graphs corresponding to the three dimensional platonic solids can be found in Tab.(III).

All these examples indicate three different tendencies for the maximal EoF:

1. $E_{\max }$ decreases with the number of adjacent vertices.

\begin{tabular}{|l|c|c|c|c|}
\hline platonic solid & $E_{\max }$ & $N_{a}$ & $N$ & $E_{0}$ \\
\hline tetrahedron & 19.74 & 3 & 4 & $\frac{1}{\sqrt{2}}$ \\
cube & 19.74 & 3 & 8 & $\frac{1}{\sqrt{2}}$ \\
dodecahedron & 11.12 & 3 & 20 & $\frac{1}{30}(12+5 \sqrt{2}+2 \sqrt{5})$ \\
octahedron & 10.75 & 4 & 6 & $\frac{1}{6}(3+\sqrt{3})$ \\
icosahedron & 5.37 & 5 & 12 & $\frac{1}{\sqrt{5}}+\frac{1}{\sqrt{6}}$ \\
\hline
\end{tabular}

TABLE II: Maximal amount of nearest neighbor entanglement $E_{\text {max }}$ (measured in units of $10^{-2}$ ebits) and the respective ground state energy (minimal EPR-uncertainty) $E_{0}$ for the five platonic solids. $N_{a}$ is the number of adjacent vertices and $N$ the total number of nodes.

2. $E_{\max }$ decreases with the total number of vertices.

3. $E_{\max }$ is suppressed in loops with an odd number of vertices, which give rise to additional frustration.

Let us now to proceed to prove our main result. We denote by $\Gamma$ and $\gamma \mathrm{CM}$ of the global state and the reduced density operator for the modes $k, l$ in whose entanglement we are interested. The first CM must fulfill

$$
\Gamma=\frac{1}{|G|} \sum_{g \in G}\left(T_{g} \oplus T_{g}\right) \Gamma\left(T_{g} \oplus T_{g}\right)^{T} .
$$

The CM $\gamma$ of a two-mode subsystem can always be written, up to local symplectic transformations $S_{1,2}$, in the standard form [3]

$$
\left(S_{1} \oplus S_{2}\right) \gamma\left(S_{1} \oplus S_{2}\right)^{T}=\left(\begin{array}{cc}
n_{A} & k_{q} \\
k_{q} & n_{B}
\end{array}\right) \oplus\left(\begin{array}{cc}
n_{A} & k_{p} \\
k_{p} & n_{B}
\end{array}\right) .
$$

The fact that $G$ contains by assumption an element which maps $k \leftrightarrow l$ immediately implies that $n_{A}=n_{B}$ and $S_{1}=$ $S_{2}=S$. Hence, given a global $\mathrm{CM}$ we can always find another one given by $\left(\bigoplus_{i=1}^{N} S\right) \Gamma\left(\bigoplus_{i=1}^{N} S\right)^{T}$, which is also $G$-symmetric, and such that $\gamma$ has the standard form (15) with $n_{A}=n_{B}=: n$.

We are interested in maximizing the EoF of $\gamma$. Since $n_{A}=n_{B}$ we can use the results of [6], which show that this quantity is given by $E_{F}(\Delta)$, where the function $E_{F}$ has been given in (2), which is a monotonically decreasing function of the so-called EPR-uncertainty $\Delta$. Thus, maximizing the EoF is equivalent to minimizing $\Delta$. This last quantity is a highly nonlinear function of the parameters $n, k_{q}, k_{p}$, and thus minimizing it with respect to all possible global $\Gamma$ looks as a very daunting task. In order to overcome this problem, the trick is to linearize the expression of $\Delta$ by including an extra maximization in the problem, i.e. writing

$$
\begin{aligned}
\Delta & =\inf _{s>0} \operatorname{tr}\left[\gamma\left(s h_{+}^{(k, l)} \oplus \frac{1}{s} h_{-}^{(k, l)}\right)\right] \\
& =\inf _{s>0} \operatorname{tr}\left[\Gamma\left(s H_{+} \oplus \frac{1}{s} H_{-}\right)\right] .
\end{aligned}
$$

In the last step we have used that $\gamma$ is the reduced $\mathrm{CM}$ of $\Gamma$, and Eq.(14). 
We show now that $\Gamma$ must correspond to a pure state. If a $\mathrm{CM} \Gamma_{m}$ corresponds to a $G$-symmetric mixed state, it can always be decomposed into a $G$-symmetric pure state $\mathrm{CM} \Gamma_{p}$ and a $G$-symmetric matrix $M \geq 0$ via $\Gamma_{m}=\Gamma_{p}+$ $M[15$. This decomposition can be interpreted as adding classical Gaussian noise to $\Gamma_{p}$ [4]. Since this will certainly not increase the entanglement between any two modes of the system, maximal entanglement will be attained if the overall CM $\Gamma$ corresponds to a pure state.

We can thus exploit the fact that every pure state CM can be written as

$$
\Gamma=\left(\begin{array}{cc}
X & X Y \\
Y X & Y X Y+X^{-1}
\end{array}\right),
$$

with $X>0$ and $Y=Y^{T}$ [7]. Hence, the EPRuncertainty (16a) becomes

$$
\Delta=\inf _{s>0} s \operatorname{tr}\left[X H_{+}\right]+\frac{1}{s} \operatorname{tr}\left[\left(X^{-1}+Y X Y\right) H_{-}\right],
$$

Maximizing the entanglement means minimizing $\Delta$ with respect to $X$ and $Y$ under the constraint that they parameterize a $G$-symmetric CM. We can, however, drop this constraint since the symmetry of $H_{ \pm}$will force the optimal $\Gamma_{0}$ to have the right symmetry. Moreover, $\Gamma_{0}$ will be the ground state corresponding to the Hamiltonian matrix $H=H_{+} \oplus H_{-}$since

$$
\begin{aligned}
\Delta_{0} & =\inf _{X, Y} \Delta=\inf _{X} \operatorname{tr}\left[X H_{+}\right]+\operatorname{tr}\left[X^{-1} H_{-}\right] \\
& =\inf _{\Gamma} \operatorname{tr}\left[\Gamma\left(H_{+} \oplus H_{-}\right)\right]
\end{aligned}
$$

where we have first set $Y=0$, since $\operatorname{tr}\left[Y X Y H_{-}\right] \geq 0$ and then incorporated the infimum over $s$ into that over $X$. This completes the proof of our main result, since $\Delta_{0}$ is by Eqs. (420) equal to the ground state energy $E_{0}$ of the Hamiltonian in Eq.(11).

By imposing less restrictions on the symmetry group than requiring the existence of an element which interchanges $k \leftrightarrow l$, the maximal achievable EoF could grow. In the examples on chains and lattices, we implicitly imposed the translational and reflection symmetry. The same results can however be derived with only the translational symmetry. In fact, the presented proof can be extended in a straight forward manner to all symmetry groups with Abelian commutant, including those of rings and cubic lattices, without imposing reflection symmetry. The proof can be found in the appendix.

In conclusion, we have determined the maximal entanglement between two modes under the constraint that the overall system is in a Gaussian state which has some symmetry with respect to the ordering of the modes. The result was derived by linearizing the entanglement functional which permits to perform the maximization in a simple way. We find that the maximal entanglement is connected to the ground state of a particular quadratic
Hamiltonian which possesses the same symmetry as the state. The state that maximizes the EoF is precisely the ground state of such a Hamiltonian. The maximal entanglement turned out to be finite in all the discussed cases, and is even comparable to the (conjectured) values for spin $\frac{1}{2}$ systems for the case of rings $[9]$. We have shown how the entanglement decreases with the number of spatial dimensions, and how it depends on the geometry of the state. Finally, although we have concentrated here on Gaussian states, we think that similar results may be obtained for the case of qubits. In that case it is also possible to linearize the expression for the EoF [12] and in this way to relate it to the ground state energy of a specific nearest-neighbor interaction Hamiltonian, which allowed us to solve the problem for Gaussian states.

We acknowledge interesting discussions with J. Garcia Ripoll and G.Giedke. This work was supported in part by the E.C. (projects RESQ and QUPRODIS) and the Kompetenznetzwerk "Quanteninformationsverarbeitung" der Bayerischen Staatsregierung.

[1] A. Holevo, Probabilistic and statistical aspects of quantum theory (North-Holland Publishing Company, 1982).

[2] B. Julsgaard, A. Kozhekin, and E. S. Polzik, Nature 413, 400 (2000).

[3] L.-M. Duan, G. Giedke, J. I. Cirac, and P. Zoller, Phys. Rev. Lett. 84, 2722 (2000); R. Simon, Phys. Rev. Lett. 84, 2726 (2000).

[4] R. F. Werner and M. M. Wolf, Phys. Rev. Lett. 86, 3658 (2001).

[5] K. Audenaert, J. Eisert, M. B. Plenio, and R. F. Werner, Phys. Rev. A 66, 042327 (2002).

[6] G. Giedke, M. M. Wolf, O. Krüger, R. F. Werner, and J. I. Cirac, (2003), quant-ph/0304042.

[7] M. M. Wolf, G. Giedke, O. Krüger, R. F. Werner, and J. I. Cirac (2003), quant-ph/0306177.

[8] R. F. Werner, Lett. Math. Phys. 17, 359 (1989); B. Schumacher and R. F. Werner, in preparation.

[9] K. O'Connor and W. K. Wootters, Phys. Rev. A 63, 052302 (2001); W. K. Wootters, Contemp. Math. 305, 299 (2002).

[10] C. H. Bennett, D. P. DiVincenzo, J. A. Smolin, and W. K. Wootters, Phys. Rev. A 54, 3824 (1996).

[11] R. A. Horn and C. R. Johnson, Matrix Analysis (Cambridge University Press, 1987).

[12] F. Verstraete, M.M. Wolf, and J. I. Cirac, in preparation.

[13] We use $|k\rangle$ to denote the unit vector in $\mathbb{R}^{N}$ whose only nonzero component is the $k$-th. Thus, $T_{g}|k\rangle=|g(k)\rangle$.

[14] Eq. (6) is derived by exploiting that the entries of the diagonal matrix $D$ are the square roots of the eigenvalues of $\left(\sigma H \sigma^{T} H\right)$ [1].

[15] To see this, note that we can always subtract a matrix $\tilde{M} \geq 0$ from $\Gamma_{m}$ such that still $\left(\Gamma_{m}-\tilde{M}\right) \geq i \sigma$ holds. Averaging this equation over the group $G$ tells us that we can always choose $\tilde{M}$ to be invariant under $G$. Subtracting as much of a $G$-symmetric and positive semi-definite matrix as possible leads us then to the symmetric pure state $\mathrm{CM} \Gamma_{p}$. 


\section{APPENDIX: GROUPS WITH ABELIAN COMMUTANT}

In this appendix, it will be shown that the reflection symmetry need not be imposed if the commutant of the group is Abelian, as the states with maximal EoF automatically have this property. The proof is as follows. The reduced covariance matrix corresponding to modes $k$ and $l$ of a state with a symmetry that maps $g(k)=l$ is of the form

$$
\gamma=\left(\begin{array}{llll}
a & b & d & e \\
b & c & f & g \\
d & f & a & b \\
e & g & b & c
\end{array}\right)
$$

It can readily be checked that this can be brought into symmetric normal form $\tilde{\gamma}$ by local symplectic transformations of the form

$$
\tilde{\gamma}=(O \oplus \mathbf{1})(S \oplus S) \gamma(S \oplus S)^{T}\left(O^{T} \oplus \mathbf{1}\right)
$$

with $S$ symplectic and $O$ orthogonal. Suppose now that $\gamma$ and the global covariance matrix $\Gamma$ are such that $S=$ 1, which can always be done by a transformation $\Gamma \mapsto$ $\left(\oplus_{i=1}^{N} S^{\prime}\right) \Gamma\left(\oplus_{i=1}^{N} S^{\prime}\right)^{T}$. Then the EPR-uncertainty $\Delta$ is given by

$\Delta=\inf _{s>0} \operatorname{Tr}[\gamma \underbrace{\left(O^{T} \oplus \mathbf{1}\right)\left(\begin{array}{cccc}s & 0 & s & 0 \\ 0 & 1 / s & 0 & -1 / s \\ s & 0 & s & 0 \\ 0 & -1 / s & 0 & 1 / s\end{array}\right)(O \oplus \mathbf{1})}_{h}]$.

Parameterizing $O=\left(\begin{array}{cc}\cos (\theta) & -\sin (\theta) \\ \sin (\theta) & \cos (\theta)\end{array}\right)$, one gets

$$
\begin{aligned}
h_{q q} & =\left(\begin{array}{cc}
s \cos ^{2}(\theta)+\frac{\sin ^{2}(\theta)}{s} & s \cos (\theta) \\
s \cos (\theta) & s
\end{array}\right) \\
h_{q p}=h_{p q}^{T} & =\left(\begin{array}{cc}
\left(\frac{1}{s}-s\right) \cos (\theta) \sin (\theta) & -\frac{\sin (\theta)}{s} \\
-s \sin (\theta) & 0
\end{array}\right) \\
h_{p p} & =\left(\begin{array}{cc}
s \sin ^{2}(\theta)+\frac{\cos ^{2}(\theta)}{s} & -\frac{\cos (\theta)}{s} \\
-\frac{\cos (\theta)}{s} & \frac{1}{s}
\end{array}\right)
\end{aligned}
$$

Let's next extend these Hamiltonian blocks to $N$-modes by averaging over the group:

$$
H_{\alpha \beta}=\frac{1}{|G|} \sum_{g \in G} T_{g} h_{\alpha \beta} T_{g}^{T}
$$

The goal is now to calculate the sum of the symplectic eigenvalues of this Hamiltonian. As the commutant of the group was assumed to be Abelian, all $N \times N$ matrices

$$
A_{i j}=\frac{1}{|G|} \sum_{g \in G} T_{g}|i\rangle\langle j| T_{g}^{T},
$$

$(i, j) \in\{0,1\}$, commute and hence can be diagonalized simultaneously by a unitary operator (eventually complex), yielding diagonal elements $\lambda_{i j}^{\mu}$, where $(i, j) \in\{0,1\}$ and $1 \leq \mu \leq N$. Applying the same unitary transformation to the four matrices $H_{\alpha \beta}$, the complete Hamiltonian becomes a direct sum of $2 \times 2$ blocks of the form

$$
B_{\mu}=\left(\begin{array}{cc}
b_{q q}^{\mu} & b_{q p}^{\mu} \\
b_{q p}^{\mu *} & b_{p p}^{\mu}
\end{array}\right), \quad b_{\alpha \beta}^{\mu}=\sum_{i j} h_{\alpha \beta}^{i j} \lambda_{i j}^{\mu}
$$

In general, the symplectic eigenvalues of a positive operator $\Gamma$ are given by the square roots of the eigenvalues of the operator $\left(\Gamma \sigma \Gamma^{\dagger} \sigma^{T}\right)$, and the unitary operation under consideration does not change these eigenvalues. It can now readily be checked that the sum of the symplectic eigenvalues corresponding to the block $B_{\mu}$ is given by the formula

$$
\nu_{1}^{\mu}+\nu_{2}^{\mu}=\sqrt{b_{q q}^{\mu} b_{p p}^{\mu}-\left(\operatorname{Re}\left(b_{q p}^{\mu}\right)\right)^{2}} .
$$

Due to equation (24), it holds that

$$
b_{q q}^{\mu} b_{p p}^{\mu}-\left(\operatorname{Re}\left(b_{q p}^{\mu}\right)\right)^{2}=\left(\bar{\lambda}^{\mu}\right)^{T} Q \bar{\lambda}^{\mu}
$$

where

$$
\begin{aligned}
\bar{\lambda}^{\mu} & =\left(\begin{array}{c}
\lambda_{00}^{\mu} \\
\operatorname{Re}\left(\lambda_{01}^{\mu}\right) \\
\lambda_{11}^{\mu}
\end{array}\right), \\
Q & =\left(\begin{array}{ccc}
1 & 0 & 1 \\
0 & -4 & 0 \\
1 & 0 & 1
\end{array}\right)+\frac{\sin ^{2}(\theta)}{2}\left(s-\frac{1}{s}\right)^{2}\left(\begin{array}{ccc}
0 & 0 & 1 \\
0 & -2 & 0 \\
1 & 0 & 0
\end{array}\right) .
\end{aligned}
$$

The presence of the second term in $Q$ (which is not there when reflection symmetry is present) can never lead to an improvement of the entanglement, as it can only increase the symplectic eigenvalues. Indeed, it holds that

$$
\lambda_{00}^{\mu} \lambda_{11}^{\mu}-\left|\lambda_{01}^{\mu}\right|^{2} \geq 0
$$

as it is the determinant of a principal submatrix of a positive $2 N \times 2 N$ matrix $P$. ( $P$ is obtained by first applying the group symmetry to the hypothetical blocks $h_{q q}=|0\rangle\left\langle 0\left|; h_{q p}=h_{p q}^{T}=\right| 0\right\rangle\left\langle 1\left|; h_{p p}=\right| 1\right\rangle\langle 1|$ (which is manifestly positive), and then diagonalizing the four blocks by a unitary transformation.) Therefore, the optimal choice for the orthogonal matrix $O$ is to choose it equal to the identity, imposing that the optimal solution obeys the reflection symmetry. 